\title{
Developing and Implementing an Imaging Optimization Study in Pediatric Nuclear Medicine: Experience and Recommendations from an IAEA-Coordinated Research Project
}

\author{
Gian Luca Poli ${ }^{1}$, Marco Coca $^{2}$, Leonel Torres ${ }^{3}$, Frederic Fahey ${ }^{4}$, Michael Lassmann ${ }^{5}$, Claire-Louise Chapple ${ }^{6}$, \\ Peter Homolka ${ }^{7}$, and Harry Delis ${ }^{1}$ \\ ${ }^{1}$ Dosimetry and Medical Radiation Physics Section, International Atomic Energy Agency, Vienna, Austria; ${ }^{2}$ Medscan Nuclear \\ Medicine and PET/CT Center, Concepcion, Chile; ${ }^{3}$ Department of Nuclear Medicine, DIC, CENTIS, Havana, Cuba; ${ }^{4}$ Division of \\ Nuclear Medicine, Boston Children's Hospital, Boston, Massachusetts; ${ }^{5}$ Department of Nuclear Medicine, University Hospital \\ Würzburg, Würzburg, Germany; ${ }^{6}$ Newcastle upon Tyne Hospitals NHS Trust, Newcastle upon Tyne, United Kingdom; and ${ }^{7}$ Center for \\ Medical Physics and Biomedical Engineering, Medical University of Vienna, Vienna, Austria
}

See an invited perspective on this article on page 568 .

The International Atomic Energy Agency instituted a coordinated research project on the evaluation and optimization of pediatric imaging, addressing the lack of consistency in this field. The purpose was to develop and test an optimization schema for the practices of pediatric radiology and nuclear medicine. Methods: A 5 -step optimization schema was developed. Once a protocol optimization is identified, the steps are as follows: identification of the imaging situation; collection of administered-activity data and evaluation of the diagnostic image quality at baseline; comparison of baseline administered activity data with published standards or other benchmarks; identification of intervention, if necessary; and implementation of intervention and evaluation. Results: Within the coordinated research project, two sites considered optimization projects regarding nuclear medicine. In this work, renal imaging using 99mTc-dimercaptosuccinic acid (DMSA) projects are presented as examples. Site 1 acquired its standard 300 -s static $99 \mathrm{mTc}$-DMSA studies as 5-frame dynamic studies in 29 children. Frames were added to simulate different levels of administered activity. Image quality was subjectively judged on a 3-point Likert scale. A 30\% reduction in administered activity with increased imaging duration (350 s) across all age groups was shown to be acceptable. This reduction was implemented and evaluated in 31 subsequent children, yielding administered activities significantly lower than baseline (mean relative differences of $30 \%$, $37 \%$, and $38 \%$ for children aged $0-5,5-10$, and $10-15 y$, respectively). Site 2 performed a phantom study to determine the impact of lowering administered activity on image noise, finding that administered activities could be significantly lowered if longer imaging times were used. This led to a $50 \%-70 \%$ reduction from baseline with no loss in image quality. Conclusion: A dose optimization approach was applied successfully for several procedures commonly performed in pediatric nuclear medicine. Results are reported for renal cortical imaging using 99mTc-DMSA, leading to significant reductions in administered activity (and thus radiation dose). This optimization schema can be

Received Mar. 24, 2020; revision accepted Jul. 22, 2020.

For correspondence or reprints contact: Gian Luca Poli, Dosimetry and Medical Radiation Physics Section, International Atomic Energy Agency, P. O. Box 100, Vienna 1220, Austria.

E-mail: g.luca.poli@gmail.com

Published online Aug. 28, 2020.

COPYRIGHT (c) 2021 by the Society of Nuclear Medicine and Molecular Imaging. successfully implemented by nuclear medicine clinics seeking to improve their approach to imaging children.

Key Words: pediatric imaging; optimization study; dose reduction

J Nucl Med 2021; 62:570-576

DOI: $10.2967 /$ jnumed.120.244616

$\mathbf{T}$ he clinical utility of nuclear medicine imaging in children has been clearly established for many applications, including neurology, urology, orthopedics, gastroenterology, endocrinology, and oncology (1-8). However, children are a critical group of patients for exposure to ionizing radiation because of their increased radiosensitivity and longer life expectancy. For a particular examination, there can be wide variations in image quality and administered activities among different nuclear medicine departments, depending not only on the protocol being followed but also on the experience of the personnel and the suitability of the equipment. Dose optimization in nuclear medicine imaging not only is motivated by the need for continuous improvement in the quality of practices but also is a legal requirement $(9,10)$ that should be achieved through a multidisciplinary approach. In 2015, the International Atomic Energy Agency established a coordinated research project (CRP) (11) with the purpose of enhancing the potential of participating institutes to improve the efficiency of existing modalities for pediatric medical imaging. The CRP included both diagnostic radiology and nuclear medicine practices and aimed at developing and implementing optimization strategies in pediatric imaging. Institutes participating in the CRP were from 10 different International Atomic Energy Agency member states (Austria, Brazil, Chile, Cuba, Egypt, Germany, Ghana, Indonesia, United Kingdom, and United States). The Department of Nuclear Medicine, Isotopes Center, Havana, Cuba, and the Medscan Nuclear Medicine and PET/CT Center, Concepcion, Chile, specifically contributed to the nuclear medicine part of the project. One of these sites will be referred to as Site 1 and the other Site 2 .

The optimization methodology developed under this CRP was applied to children but can be equally adopted for any other 
population. Optimizing a diagnostic nuclear medicine procedure does not necessarily translate into a reduction of administered activity but, more correctly, to the administration of the most appropriate activity. The ultimate objective of medical imaging is to draw appropriate diagnostic information from the images produced. Thus, any optimization process necessarily needs to consider the resulting clinical image quality.

This paper focuses on the work done for nuclear medicine procedures and can be used as an educational tool providing practical guidance on developing an optimization study and its successful implementation, with illustrations from the work presented and the lessons learned.

\section{MATERIALS AND METHODS}

Institutes from 10 different countries participated in the CRP. The optimization schema described below was developed, and each institute agreed to implement it for either diagnostic radiology or nuclear medicine. In this paper, the version developed for nuclear medicine is described, which is very similar to the schema implemented for diagnostic radiology.

The steps followed for the optimization process are outlined in Figure 1. An initial preoptimization data collection of the local situation is followed by a comparison of these data with guidelines and existing activity reference levels to identify deviations and areas for improvement. Potential interventions identified through a multidisciplinary approach need to be translated into practice in the next step, and their efficacy verified. A new data collection after the intervention is finally performed for comparison with the baseline situation and verification of a positive result.

\section{Identification of the Imaging Situation}

The first step was to identify the procedures requiring consideration, based on the frequency for pediatric patients and potential radiation dose. Data on the number of procedures performed on children were collected in 3 age ranges: $0-5,5-10$, and 10-15 y. The institutes involved in the project identified static renal scintigraphy ( ${ }^{99 \mathrm{~m}}$ Tc-dimercaptosuccinic acid [DMSA]), dynamic renal scintigraphy ( ${ }^{99 \mathrm{~m} T c-m e r c a p t o a c e t y l t r i g l y c i n e ~[M A G 3]), ~ a n d ~ b o n e ~ s c i n t i g r a p h y ~}$ ( $99 \mathrm{~m}$ Tc-methylene diphosphonate [MDP]) as the 3 most frequent procedures in children. Figure 2 shows the distribution of pediatric studies at site 1 during 2018 and at site 2 during 2017.

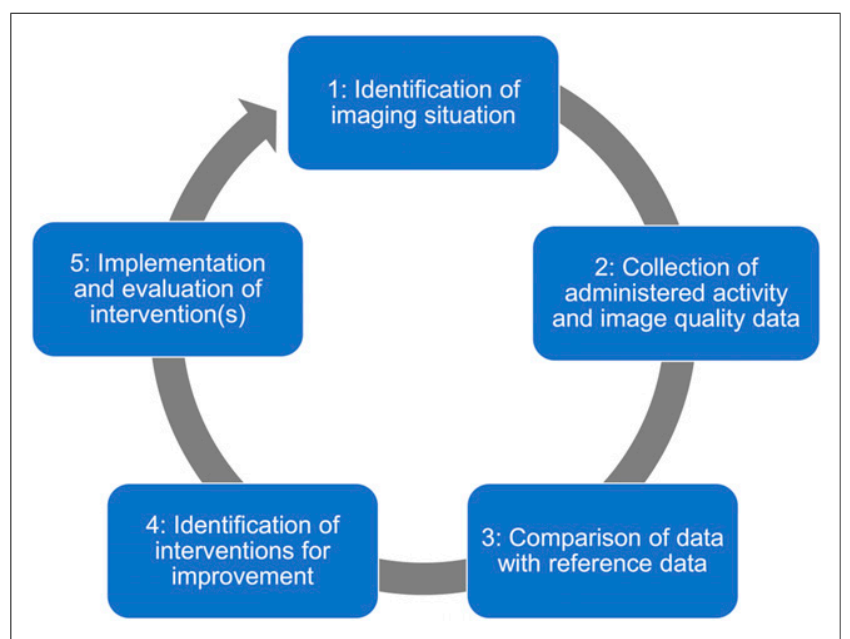

FIGURE 1. General scheme describing the optimization strategy.

\section{Collection of Administered Activity Data at Baseline}

A baseline was established by collecting administered activity data for children undergoing the procedures identified above over 9 mo. The data collected included patient age, height, weight, and sex. Activity values were collected for the syringe prepared for administration, as well as residual activity in the syringe after administration. The time of these two measurements and the time of administration were also collected to allow the calculation of the decay-corrected administered activity.

\section{Evaluation of Diagnostic Image Quality at Baseline}

Image quality was assessed both qualitatively through an image quality factor representing a 3-point Likert scale and quantitatively using phantom measurements. The image quality factor adopted for the purposes of the CRP ranged from IQ1 to IQ3: IQ1 is unacceptable image quality (could not diagnose, would request a rescan), IQ2 is borderline-acceptable image quality (can diagnose but would not welcome images of this quality), and IQ3 is acceptable image quality (can diagnose and would welcome images of this quality)

Nuclear medicine physicians' involvement was vital at this step of the methodology, highlighting the need for a multidisciplinary approach to dose optimization. Because such subjective assessments depend greatly on both the evaluator and the clinical question, they may not be directly comparable between centers.

Alternatively, physical phantoms simulating the clinical condition can be used to generate images at various noise levels with objective measurements (i.e., contrast, noise, and signal-to-noise ratio) to assess image quality. In this manner, the effect that different count levels (and thus different administered activity levels) has on image quality can be determined and compared.

\section{Comparison of Data with Reference Data}

Baseline administered activities should be compared with wellestablished references from scientific societies or international organizations. In this study, the recommended administered radiopharmaceutical activities for pediatric patients proposed in the North American Consensus Guidelines (NACG) (12) and the European Association of Nuclear Medicine (EANM) pediatric dosage card (13) were used for this purpose. This step of the process determines whether an intervention is necessary and, if so, what intervention should be considered.

\section{Identification of Interventions for Improvement}

On the basis of these assessments, a procedure can be identified as suboptimal, and an intervention can thus be determined to be necessary. However, the need for an intervention should not always be assumed. In some cases, the current practice may continue to be acceptable, or the value of a reasonable intervention may be marginal and not warranted at a particular time. However, when an intervention is deemed necessary, a proposed target optimization level is specified. This target could be defined as a set fraction of the current level or a set level relative to the published standard. In some instances, a different level may be specified for each patient size descriptor considered (i.e., patient weight, age, or other measure). However, in many cases, setting a single target level for children of all sizes may be considered appropriate and simpler to implement.

\section{Implementation and Evaluation of the Intervention}

Once an intervention is determined to be necessary and a target level is proposed, the clinical procedure is thereby amended. It may be preferable to implement the intervention gradually. All nuclear medicine personnel (physicians, technologists, medical physicists, radiopharmacists, and possibly others) must be informed that the procedure will be revised in a certain way on a certain date. All patients imaged using this protocol on or after the proposed start date will receive the modified administered activity. If it is determined that the intervention introduced a complication that was not foreseen, the clinic may decide to return to 


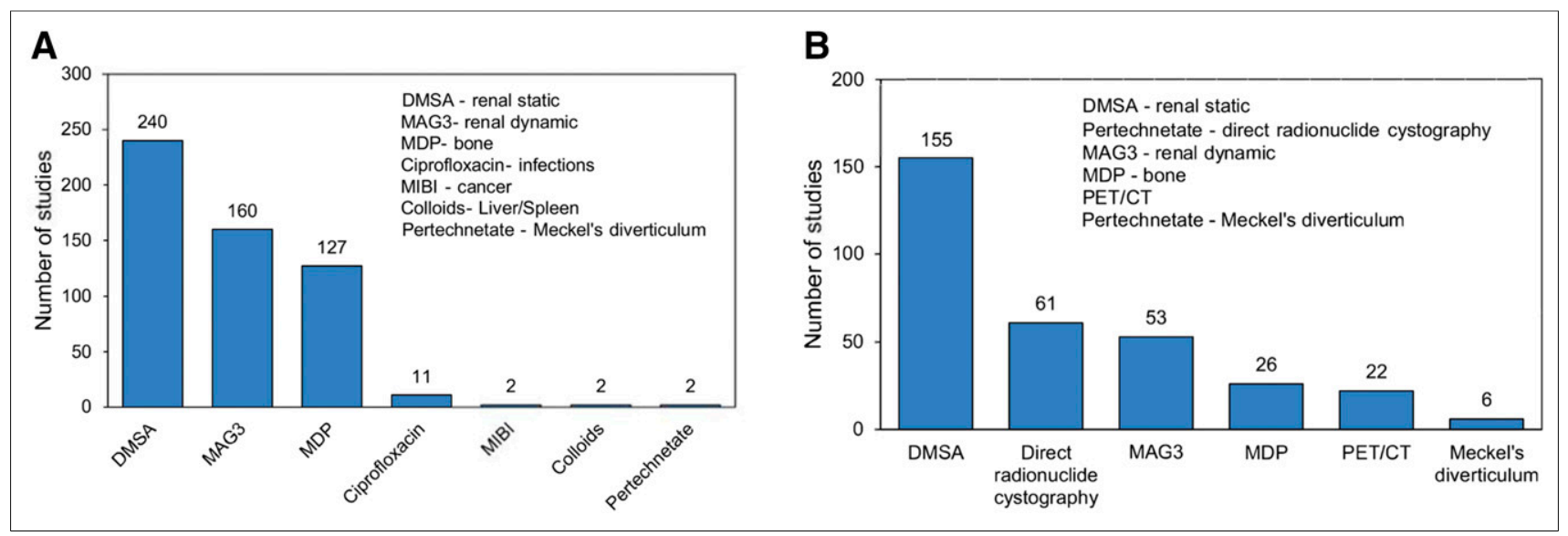

FIGURE 2. Distribution of pediatric studies at site 1 (2018) (A) and site 2 (2017) (B). MIBI = methoxyisobutylisonitrile.

the former administered activity level at any time. Otherwise, the new level should be maintained for a specified evaluation period measured either in time or number of cases (e.g., for 1 mo or for 30 cases).

Once the evaluation period is complete, the resulting patient data need to be evaluated to determine whether the proposed target levels were achieved and whether the image quality remained acceptable. For each patient, the actual administered activity should be compared with the proposed value and the previously prescribed level. These activity levels can also be compared with those prescribed by published standards. Any concerns from the clinical staff that the intervention resulted in marginal or less than adequate image quality also must be considered. If, after this evaluation, it is determined that the target level was reached without a notable reduction in clinical image quality, the new administered activity levels are adopted.

Working groups from sites 1 and 2 implemented this methodology for several nuclear medicine procedures commonly performed on children. The institutional medical committee approved this study at both sites, and the requirement to obtain informed consent was waived. Renal scintigraphy with ${ }^{99 \mathrm{~m} T c-D M S A}$ is presented here as an example since it was optimized by both groups, with the methodology being implemented using slightly different approaches. For both centers, only one dual-head $\gamma$-camera equipped with low-energy high-resolution collimators was involved in the study. The systems used were Spirit DH-V (Mediso) and Sophy DST-XLi (SMV) for sites 1 and 2, respectively.

\section{RESULTS}

\section{Site 1}

Since the 3 most common procedures at site 1 were ${ }^{99 m}$ Tc-DMSA

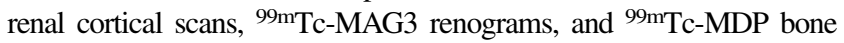
scans (Fig. 2A), the optimization process was focused on these. The results for renal cortical imaging using ${ }^{99 \mathrm{~m}} \mathrm{Tc}$-DMSA are presented here as an example. The baseline administered activities for ${ }^{99 \mathrm{~m}} \mathrm{Tc}-$ DMSA were calculated as a fraction of the activity for a 70-kg adult (185 MBq), based on body surface area as recommended in Operational Guidance on Hospital Radiopharmacy (14). The baseline situation was assessed by reviewing data for 29 patients (aged 6 mo to $18 \mathrm{y}$ ), noting age, weight, height, and sex for each. The activity at the time of administration was calculated as the difference between the decaycorrected activities assayed in the preinjection syringe and the residual activity after administration. These baseline levels were significantly higher than those recommended by the NACG (12) and the EANM pediatric dosage card (Fig. 3) (13). Three age groups, 0-5, 5-10, and
10-15 y, were considered. The administered activities were, on average, $149 \%$ (0-5 y), 103\% (5-10 y), and 57\% (10-15 y) higher than the NACG-recommended values. As a result, it is expected that similar increments apply to the equivalent dose received by the critical organs (kidneys) and to the effective dose.

The adopted optimization strategy was to acquire a dynamic scan (five 1-min frames) rather than static 5-min scan to simulate different levels of administered activity for 45 patients (15 in each age group). Summing these 5 frames provided an image identical to the baseline protocol using a 5-min static acquisition. Summing 2, 3, or 4 frames of the dynamic study simulated the patient receiving an administered activity of $40 \%, 60 \%$, or $80 \%$ of the baseline activity, respectively. In total, 180 images (4 images each for the 45 patients) were randomly presented to the nuclear medicine physicians in charge of the diagnostic evaluation. The physicians' diagnostic response and IQ index for $40 \%$, $60 \%$, and $80 \%$ of the baseline activity were compared with $100 \%$ (baseline) activity. The images simulating the $40 \%$ activity level were judged adequate for quantification, albeit very noisy, which in some cases led to interobserver variations in clinical interpretation. Images simulating the $60 \%$ activity level showed no appreciable difference between patients $0-5 \mathrm{y}$ old and patients $5-10 \mathrm{y}$ old, whereas images simulating the $80 \%$ activity level were considered to be of the same diagnostic image quality as the baseline activity for all age groups.

For this reason, an initial target reduction level corresponding to $70 \%$ of the baseline administered activity was set for children of all ages and was considered an acceptable compromise by the clinicians. A single reduction level across all age groups was decided to be more easily implemented operationally. The same weighting factors as those used during the preoptimization phase and recommended by the International Atomic Energy Agency in 2008 (14) were used, but with a recommended activity of $129.5 \mathrm{MBq}$ instead of $185 \mathrm{MBq}$ for a $70-\mathrm{kg}$ adult. However, the scan duration was increased from 300 to $350 \mathrm{~s}$ as part of a conservative approach to warrant adequate image quality for patients older than $10 \mathrm{y}$. It was also agreed to apply filtering techniques to reduce noise in images with borderline count statistics (Tera-Tomo 2D Planar Image Enhancement module, Interview XP 3.00.053.0000; Mediso Medical Imaging Systems).

After introduction of the optimization intervention, data for 31 pediatric patients were collected for the following evaluation. The acquired images were considered by the clinicians to be of sufficient image quality and adequate for a sound diagnosis in all cases. Although the administered activities were still above the 


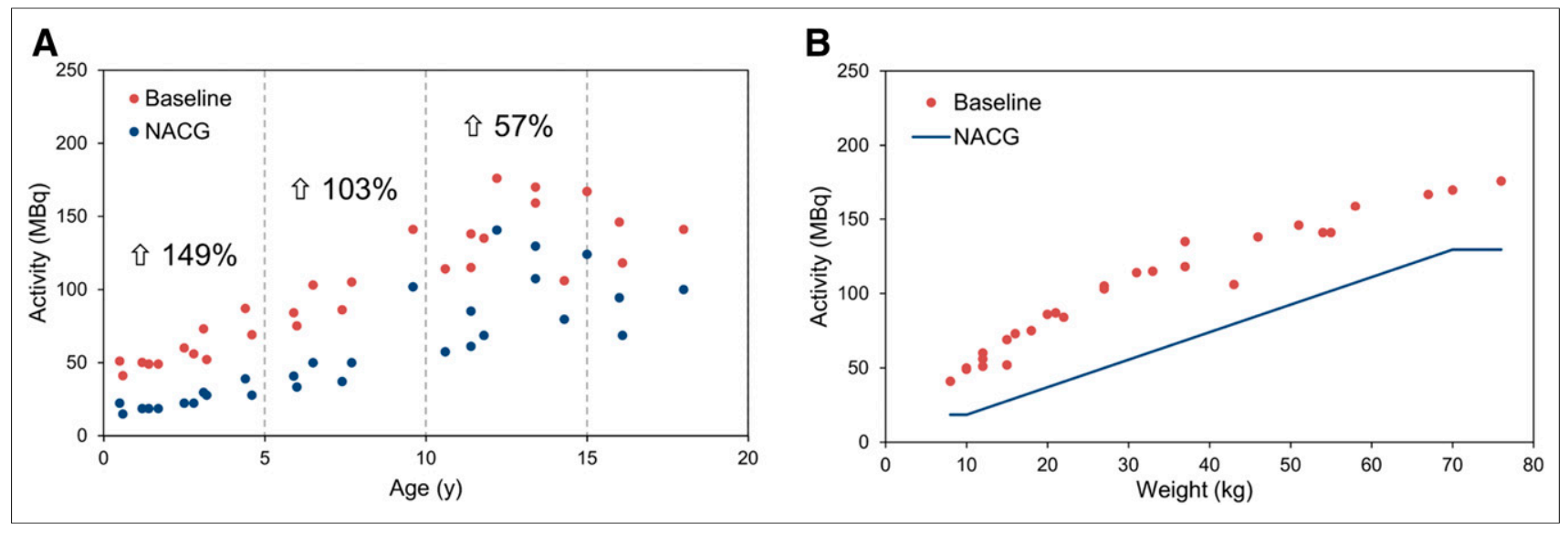

FIGURE 3. (A) Activities administered for ${ }^{99 m T c-D M S A}$ renal cortical scans at baseline and NACG-recommended activities for same patients. Average relative difference compared with NACG-recommended values is reported for each age group. (B) Same data reported as function of weight.

NACG recommendations for all patients, these activities were significantly lower than the baseline values. The mean relative differences from the NACG recommendations decreased to $75 \%$, $38 \%$, and $10 \%$ for children $0-5,5-10$, and $10-15$ y old, respectively (Fig. 4A). The considerable reduction in administered activities allowed, especially for larger children, better compliance with international recommendations (Fig. 4B).

The approach followed in this department represents a graded optimization and was agreed upon-and considered to be acceptable by-the clinicians. This approach could potentially be followed by further interventions after an adjustment period. For example, the results shown in Figure 4 suggest that a greater reduction in administered activity might be possible for smaller patients. Regarding the other procedures considered, the optimization process for ${ }^{99 \mathrm{~m}} \mathrm{Tc}-\mathrm{MDP}$ bone scans and ${ }^{99 \mathrm{~m} T c-M A G 3}$ renal studies resulted in an average reduction in administered activity by $22 \%$ and $27 \%$, respectively.

\section{Site 2}

Site 2 sought to optimize its pediatric nuclear medicine practice regarding 4 procedures: renal cortical imaging using ${ }^{99 \mathrm{~m}} \mathrm{Tc}-\mathrm{DMSA}$, renal functional imaging using ${ }^{99 \mathrm{~m}} \mathrm{Tc}-\mathrm{MAG} 3$, bone imaging using ${ }^{99} \mathrm{~m}$ Tc-MDP, and PET/CT imaging using ${ }^{18} \mathrm{~F}-\mathrm{FDG}$. The choice of the 3 planar procedures was based on a review of the number of pediatric nuclear medicine studies performed in the previous year (Fig. 2B). During that time, about $50 \%$ of the pediatric examinations performed were ${ }^{99 \mathrm{~m}} \mathrm{Tc}$-DMSA studies. The second most common procedure was direct radionuclide cystography looking for urinary reflux. Since this procedure is less amenable to the optimization approach and the resulting radiation dose is already quite low (15), the next two most common procedures were considered: ${ }^{99 \mathrm{~m} T c-M A G 3}$ renal imaging and ${ }^{99} \mathrm{~m}$ Tc-MDP bone imaging. As with site 1, this paper describes the approach for optimizing ${ }^{99 \mathrm{~m} T c-D M S A}$ planar imaging in children as an example. In total, 155 patients were examined using $99 \mathrm{~m} \mathrm{Tc}-$ DMSA in 2017, and the breakdown by age group was 100,24 , and 31 for children $0-5,5-10$, and $10-15$ y old, respectively.

Before optimization, the baseline dosing schema for 1-, 5-, and 10-y-old patients was based on an internal standard leading to respective values of 100,125 , and $155 \mathrm{MBq}$, corresponding to respective activities $550 \%, 378 \%$, and $310 \%$ higher than those recommended by EANM for the corresponding patient ages. To evaluate the potential impact of dose optimization on image
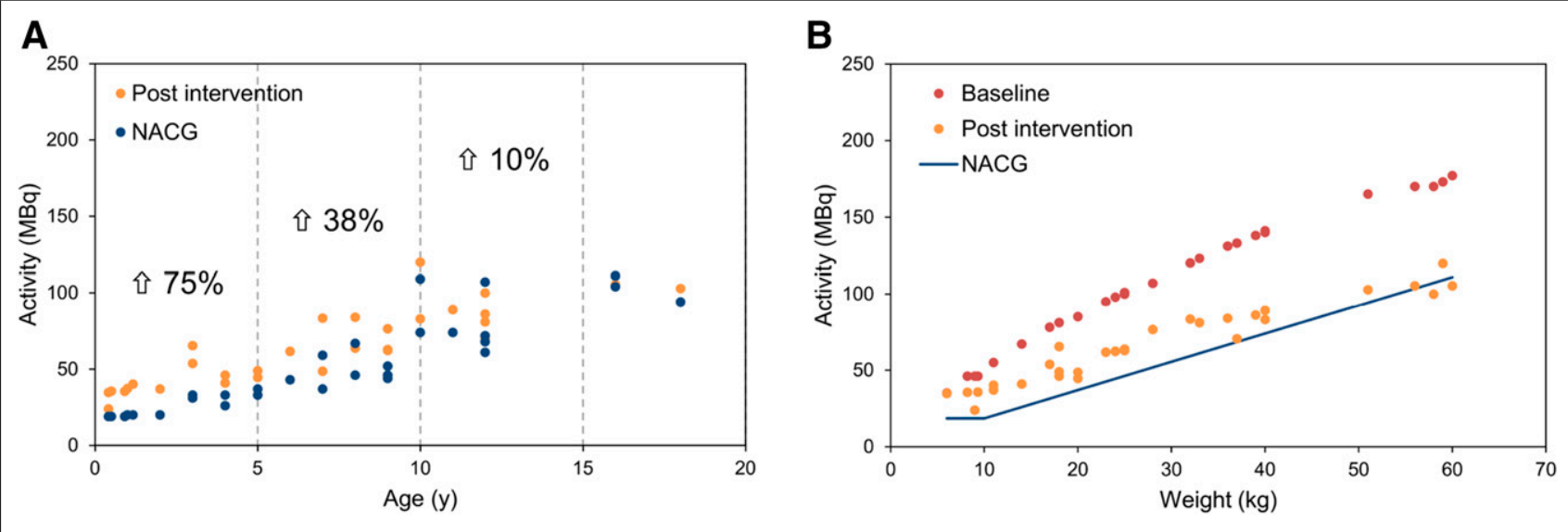

FIGURE 4. Activities administered to pediatric patients for ${ }^{99 m T c-D M S A ~ r e n a l ~ c o r t i c a l ~ s c a n s ~ a f t e r ~ i n t r o d u c t i o n ~ o f ~ o p t i m i z a t i o n ~ i n t e r v e n t i o n . ~(A) ~}$ Comparison, as function of age, with NACG-recommended activities for same patients. Average relative difference compared with NACG-recommended values is reported for each age group. (B) Comparison, as function of weight, with activities that would have been administered at baseline and NACG-recommended values for same patients. 
quality, a phantom experiment was performed. Cylindric polyethylene vials of $24.2,44.3$, and $66.3 \mathrm{~mL}$ were used to simulate the kidneys of a 1-, 5-, and 10-y-old child, respectively $(16,17)$. For each age, 2 cylinders were filled with water mixed with basically equal activity of ${ }^{99 \mathrm{~m}} \mathrm{Tc}-\mathrm{NaTcO}_{4}$ and placed within a water-filled cylindric tomographic phantom (21.6- $\mathrm{cm}$ inner diameter, 18.6-cm inner height) to simulate renal imaging. The amount of activity placed in each simulated kidney assumed that $40 \%$ of the administered activity distributes to the renal cortex at $4 \mathrm{~h}$ after injection (18), given that the acquisition start time in clinical practice is $3 \mathrm{~h}$ after administration. Since the clinical ${ }^{99 \mathrm{~m}}$ Tc-DMSA scans were acquired for $300 \mathrm{~s}$, the noise level for that acquisition time was considered a baseline value. The noise level is given by

$$
N=\frac{100 \%}{\sqrt{\frac{\text { total counts }}{\text { number of pixels }}}},
$$

where "total counts" and "number of pixels" have to be considered in the whole image. The clinical baseline of 300 kilocounts per image is considered acceptable according to international guidelines (2) and corresponds to a noise level, $N$, of $23 \%$. A noise level of $25 \%$ was also considered.

In Figure 5, the baseline administered activity levels for 3 ages is compared with the simulated value for noise levels of $23 \%$ and $25 \%$, as well as with the level recommended by the EANM and a level $50 \%$ higher than that recommended.

For the 10-y-old, the level 50\% higher than the EANM recommendation is consistent with noise levels of $23 \%$ and $25 \%$, respectively. A reduction of administered activity leading to noise levels of $23 \%$ and $25 \%$ yielded activity levels comparable to the median values for Europe and Latin America from the international survey of the Nuclear Medicine Global Initiative (19). Using this level for the 10-y-old, we determined that 300 kilocounts could still be acquired in about $5 \mathrm{~min}$. However, reaching the same level for the 5-y-old and the 1-y-old would take longer, approximately 8 and $16 \mathrm{~min}$, respectively.

The proposed target was an administered activity ranging from the EANM-recommended value and the same value increased by $50 \%$. The highest value of this range corresponded to reductions of $72 \%, 60 \%$, and $52 \%$ for the $1-, 5-$, and 10 -y-old children, respectively. This is likely to be only the first of a series of optimization steps; further reduction may be realized in the future.

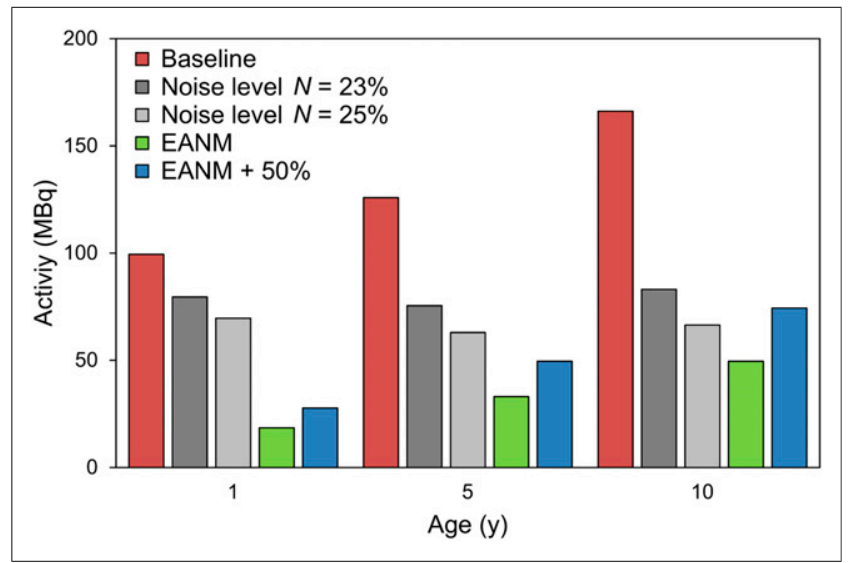

FIGURE 5. Baseline administered activity level for 3 example ages, simulated activity value corresponding to noise level of $23 \%$ and $25 \%$, activity recommended by EANM, and same value increased by $50 \%$.
After implementation of these new levels, the assayed administered activities of 20 patients were compared with both the recommended values and the values for the 18 patients before the implementation, that is, the baseline (Figs. 6A and 6B vs. age and weight). Figure 6C shows the relative difference in administered activities between baseline and postimplementation patients, compared with the EANM-recommended values. Image quality was evaluated - and the adequate amount of administered activity was assessed-by nuclear medicine physicians with the support of medical physicists and technologists involved in the optimization process.

Although the target range allowed for administered activities $50 \%$ higher than values suggested by the EANM, the actual levels of administered activity tended to be closer to the recommended
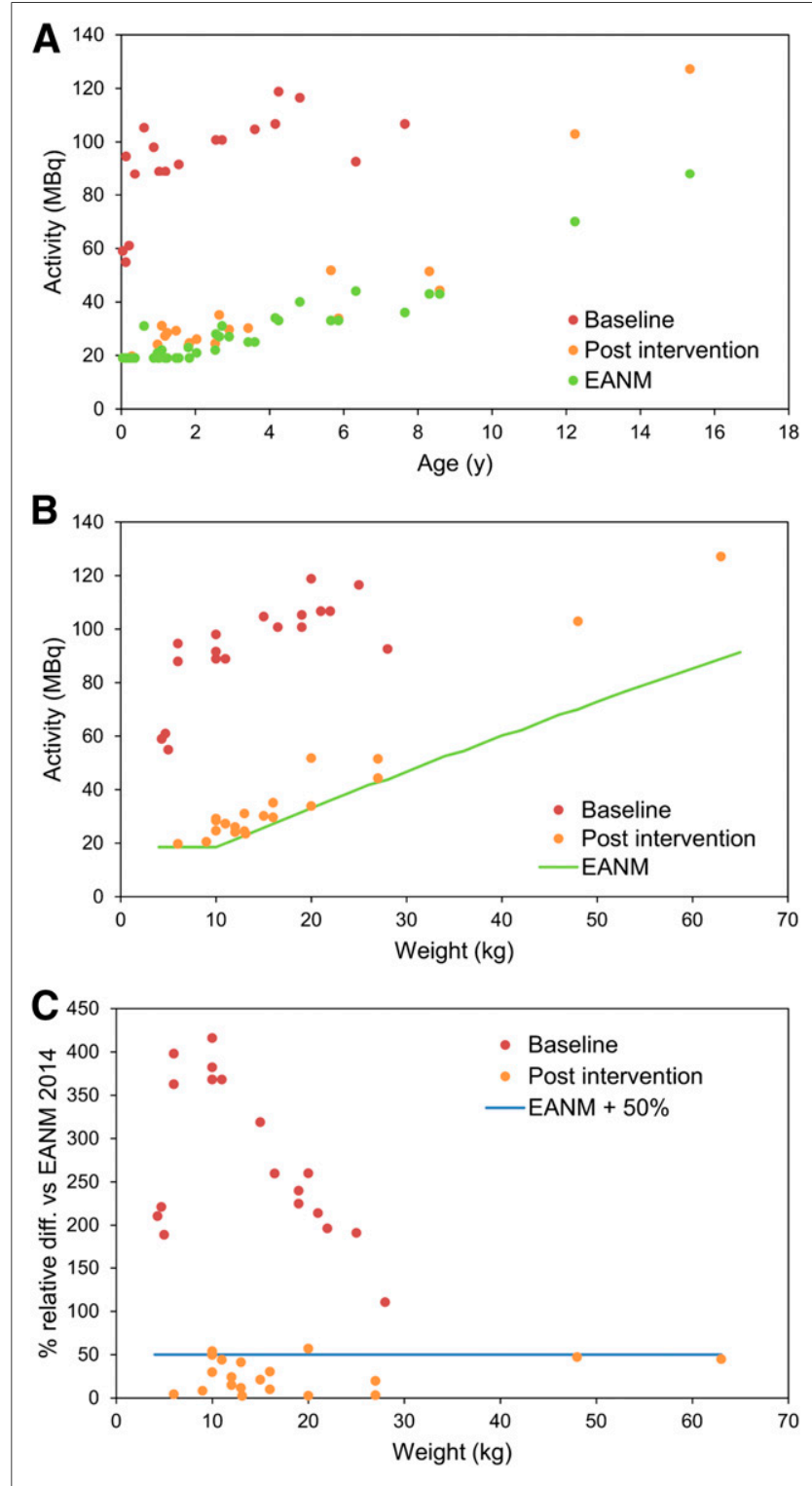

FIGURE 6. (A and B) Activities administered to pediatric patients for 99mTc-DMSA renal cortical scans at baseline, after introduction of optimization intervention, and EANM-recommended activities for same patients as function of age (A) and weight (B). (C) Relative difference in administered activities at baseline and after introduction of optimization intervention compared with EANM-recommended values. 
TABLE 1

Administered Activities for 3 Example Ages for 99mTc-DMSA Projects at Sites 1 and 2 at Baseline and After Optimization: Comparison to Recommended Values for EANM Pediatric Dosage Card and NACG

\begin{tabular}{cccccccc}
\hline Age $(\mathrm{y})$ & Weight $(\mathrm{kg})$ & EANM & NACG & Site 1 BL & Site 1 Opt & Site 2 BL & Site 2 Opt \\
\hline 1 & 10 & 18.5 & 18.5 & 50 & 35 & 100 & 28 \\
5 & 20 & 33 & 37 & 85 & 60 & 125 & 50 \\
10 & 32 & 50 & 59 & 120 & 84 & 155 & 74
\end{tabular}

$\mathrm{BL}=$ baseline; Opt $=$ after optimization

Data are megabecquerels.

levels. Since 300-kilocount images and a $23 \%$ noise level were retained, there was no reduction in image quality. For the other procedures considered, ${ }^{99 \mathrm{~m}} \mathrm{Tc}-\mathrm{MDP}$ bone scans and ${ }^{99 \mathrm{~m}} \mathrm{Tc}-\mathrm{MAG} 3$ renal studies, the optimization process resulted in average reductions of $20 \%$ and $64 \%$, respectively, in the administered activity.

\section{DISCUSSION}

As examples of the optimization schema described in this report, we have presented projects using ${ }^{99 \mathrm{~m}} \mathrm{Tc}$-DMSA for renal cortical planar imaging at two medical centers. This examination had the highest impact on dose reduction in children because of the large number of procedures performed yearly and the reduction in administered activities that could be achieved while maintaining adequate diagnostic quality. The target reductions of administered activity were $30 \%$ for site 1 and 52\%-73\% for site 2. For the 5-y-old, the target administered activities are $82 \%$ and $50 \%$ higher than the EANM-recommended value and $62 \%$ and $35 \%$ higher than the NACG-recommended value for sites 1 and 2 , respectively. Thus, there is still room for improvement, and further reductions are expected in the future. The optimization exercises described in this work are most likely the first steps in an iterative optimization process, as illustrated in Figure 1.

Although the general outline as described in the Materials and Methods section was followed in both projects, the implementation approach varied between the two sites. The baseline conditions were substantially different when the administered activities were initially considerably higher at site 2 than at site 1 . The age of the equipment between the two institutes also varied.

For both sites, only one $\gamma$-camera was available and involved in this study, thus simplifying the optimization process. If multiple scanner types with different collimators are used, the differences in performance of the systems should be considered. A scanner with lower sensitivity, for example, might require a higher injected activity or longer scan time.

Patient demographics, clinical conditions, and referral patterns most likely differ between the regions where the two institutes are located. Clinical staff expectations about the required image quality for a clinical task may also vary greatly between institutes. Lastly, each institute has its own operational considerations that affect how modifications to the clinical practice may best be realized.

The optimization process must be performed by a multidisciplinary working group, with the nuclear medicine physician being responsible for evaluating image quality and assessing the adequacy of the administered activity. Basic quantitative metrics (such as contrast and image noise) can complement evaluation of image quality during selection of activity levels providing diagnostic information. At both sites, subjective grading of clinical quality by expert observers was used, and the administered activity that optimized image quality was determined. At site 2, a phantom evaluation relying on an objective noise index was also used.

Although the two projects led to a substantial reduction in administered activity (Table 1), both also proposed a longer scan duration. Longer imaging times can be problematic when small children are imaged, because of the potential for more patient motion. For ${ }^{99 \mathrm{~m}} \mathrm{Tc}-\mathrm{DMSA}$ imaging, most patients tend to be under $5 \mathrm{y}$ old. If the imaging time is much longer than $5 \mathrm{~min}$, the use of sedation and, in some cases, anesthesia may be necessary to successfully complete the study. Therefore, even if the optimization project supports the potential for clinically adequate imaging with fewer total counts, some clinics may opt for a faster scan time in small children rather than lower administered activity.

\section{CONCLUSION}

As part of an International Atomic Energy Agency CRP, an optimization schema was developed specifically for pediatric radiology and nuclear medicine. This approach was applied successfully at two clinical sites for several procedures commonly performed in pediatric nuclear medicine. As an example, optimization of renal cortical ${ }^{99 \mathrm{~m}} \mathrm{Tc}-\mathrm{DMSA}$ imaging at these two sites led to significant reductions in administered activity (and thereby absorbed radiation dose) of between $30 \%$ and $70 \%$. Therefore, this optimization schema can be successfully implemented at practically any nuclear medicine clinic seeking to improve its approach to imaging children.

\section{DISCLOSURE}

No potential conflict of interest relevant to this article was reported.

\section{KEY POINTS}

QUESTION: Can suboptimal diagnostic procedures be identified and optimized, leading to a reduction in administered activity while maintaining an image quality sufficient for a sound diagnosis?

PERTINENT FINDINGS: An optimization strategy that was developed and applied to different pediatric nuclear medicine procedures resulted in significant reductions in administered activity (and thus radiation dose) of between $30 \%$ and $70 \%$ and no loss of image quality.

IMPLICATIONS FOR PATIENT CARE: Doses to pediatric patients can be reduced through the optimization methodology. 


\section{REFERENCES}

1. Piepsz A, Colarinha P, Gordon I, et al. Guidelines for glomerular filtration rate determination in children. Eur J Nucl Med. 2001;28:BP31-BP36.

2. Piepsz A, Colarinha P, Gordon I, et al. Guidelines for ${ }^{99 \mathrm{~m}} \mathrm{Tc}$-DMSA scintigraphy in children. European Association of Nuclear Medicine website. https://eanm.org/ publications/guidelines/gl_paed_dmsa_scin.pdf. Published October 3, 2009. Accessed November 30, 2020.

3. Stauss J, Franzius C, Pfluger T, et al. Guidelines for ${ }^{18}$ F-FDG PET and PET-CT imaging in paediatric oncology. Eur J Nucl Med Mol Imaging. 2008;35:15811588.

4. Fettich J, Colarinha P, Fischer S, et al. Guidelines for direct radionuclide cystography in children. Eur J Nucl Med Mol Imaging. 2003;30:B39-B44.

5. Gordon I, Piepsz A, Sixt R. Guidelines for standard and diuretic renogram in children. Eur J Nucl Med Mol Imaging. 2011;38:1175-1188.

6. Hahn K, Fischer S, Colarinha P, et al. Guidelines for bone scintigraphy in children. Eur J Nucl Med. 2001;28:BP42-BP47.

7. Mandell GA, Eggli DF, Gilday DL, et al. Society of Nuclear Medicine procedure guideline for renal cortical scintigraphy in children: version 3.0. Society of Nuclear Medicine and Molecular Imaging website. http://s3.amazonaws.com/ rdcms-snmmi/files/production/public/docs/pg_ch32_0403.pdf. Published June 20, 2003. Accessed November 30, 2020.

8. Shulkin BL, Mandell GA, Cooper JA, et al. Procedure guideline for diuretic renography in children 3.0. J Nucl Med Technol. 2008;36:162-168.

9. Radiation Protection and Safety of Radiation Sources: International Basic Safety Standards-General Safety Requirements Part 3. International Atomic Energy Agency; 2015.
10. European Union. Council directive 2013/59/Euratom "on basic safety standards for protection against the dangers arising from exposure to ionising radiation." J Eur Union. 2014;57.

11. Coordinated research activities. International Atomic Energy Agency website. https://www.iaea.org/services/coordinated-research-activities. Accessed November $30,2020$.

12. Treves ST, Gelfand MJ, Fahey FH, Parisi MT. 2016 update of the North American Consensus Guidelines for pediatric administered radiopharmaceutical activities. J Nucl Med. 2016;57(12):15N-18N.

13. Lassmann M, Biassoni L, Monsieurs M, et al. The new EANM paediatric dosage card. Eur J Nucl Med Mol Imaging. 2008;35:1748.

14. Operational Guidance on Hospital Radiopharmacy. International Atomic Energy Agency; 2008.

15. Ward VL, Strauss KJ, Barnewolt CE, et al. Pediatric radiation exposure and effective dose reduction during voiding cystourethrography. Radiology. 2008;249:1002-1009.

16. Tran-Gia J, Schlogl S, Lassmann M. Design and fabrication of kidney phantoms for internal radiation dosimetry using 3D printing technology. J Nucl Med. 2016; 57:1998-2005

17. Bouchet LG, Bolch WE, Blanco HP, et al. MIRD pamphlet no 19: absorbed fractions and radionuclide $\mathrm{S}$ values for six age-dependent multiregion models of the kidney. J Nucl Med. 2003;44:1113-1147.

18. Treves ST, Falone AE, Fahey FH. Pediatric nuclear medicine and radiation dose. Semin Nucl Med. 2014;44:202-209.

19. Fahey FH, Bom HH, Chiti A, et al. Standardization of administered activities in pediatric nuclear medicine: a report of the first nuclear medicine global initiative project, part 1-statement of the issue and a review of available resources. $\mathrm{J} \mathrm{Nucl}$ Med. 2015;56:646-651. 